had cause to regret using the lancet. He says: "To me it is simply an inflammation, and $I$ would use rapidly, as early as possible, the means which, in the practice of half a ceitury, I have found safe and most efficient in allaying inflammation. Well, here is my patient. He had a chill hours ago ; he had some pain in his side, some cough, and he feels very weak; his face is flushed, he is hot and thirsty, his pulse over a hundred and the thermometer rises to $103^{\circ}$; he breathes scarcely at all through the most of one lung; there is a little blood, and viscous fluid, slightly rustcolored, in the basin by the bedside; he knows how he got sick; he ' was down in the iron ore shaft all yesterday at work, came up in the evening to come home, and being in a perspiration, soon, in facing the wind, cooled off too suddenly.' I propose to bleed him, but he objects on account of his great weakness. I tie up his arm, and bleed him twenty ounces, or perhaps he feels faint by the loss of less than that. He expresses himself as being relieved somewhat of his oppression, he can lie more comfortably, he feels relieved of the fulness in the chest, but still he has a little pain in the side, or in the breast, just above the nipple. I order him one-quarter grain of sulphate of morphia; direct that a towel be wrung out of cold water, ice-water, if convenient, be applied over the affected part, to be changed frequently, or instead, a bladder of ice and water. Then ordering a mixture which contains some spirits of nitre, with about the third of a grain of morphia to a teaspoonful every two or three hours, I leave him with an express direction not to worry him by any offer of food. Next day, or even the same day, perhaps, if I find that he is not entirely relieved of the pain, and is still expectorating viscid sputa, I bleed him again freely, and if need be, cup him, and after that he is generally safe. Some one will say, 'that may do very well for your strong, iron-ore diggers, but it would prove fatal to our delicate patients.' Perhaps so, but in my experience, $I$ have found that the weak and frail need to have the same means used to allay a toothache, or a sciatica, a pleurisy or a rheumatism, as the robust. 'They bear the remedies to cure an ague as well, and need them as greatly as the strongest. I have often bled the infant strangling with croup, with immediate relief; weak women with pleurisy or pneumonia, old men, and aged, very aged women, to relieve affections of the brain, or avert a paralysis."

This is the method of treatment which Dr. Corson has practised for some fifty years, and I believe it to be much nearer right than the practice now so generally adopted.

In conclusion, I would say that I would by no means advocate or practise venesection simply because a patient had pneumonia, pleurisy, peritonitis, or meningitis, but $I$ would use it in those cases only in which $I$ considered it safe, and better adapted than any other measure for the recovery of the patient. Its use requires experience, judgment, and discrimination, but I do believe that the weakest of our patients with primary pneumonia will better bear moderate depletion, than the most robust will bear assiduous feeding and stimulation.

After venesection, I would give to such of the more robust patients as $I$ thought required it, small doses of tartar emetic, carefully watching its effects, but those who required it would be few. In some of the less robust cases, I would give small doses of ipe- cac or Dover's powder, and in all I would, until infiltration became complete, if the cuse arrived at that stage, administer opium in sufficient doses to tranquilize the nervous system, and procure sleep.

Milk I would give after the first day or two, if the patient did not object, and before that if he requested it, and more nourishing food as soon as he asked for it, but if $I$ found indications of exhaustion coming on, and had reason to believe that he could digest and assimilate food, I should urge him to try its effect before that. If the temperature was excessive, and was not reduced by venesection, I should try to reduce it by cold compresses, or quinia, or both, and I would give my patient stimulants as soon as I felt satisfied that real exhaustion was approaching, with the first indications of failure of the heart, but I would not commence the early use of stimulants to prevent the exhaustion that might occur later in the case.

I will not mention other details; they are of less importance, and about which there is less diversity of opinion. In the interest of our patients I sincerely hope that this paper may be the means of inducing some one or more of my brother practitioners to investigate and test for themselves this powerful and ton much neglected remedy, and if suitable cases are selected and the remedy used according to correct principles, I feel certain that they will learn to highly value the lancet, and to rely upon it in many cases of inflammatory disease. ${ }^{2}$

\section{THE STAINING OF THE BACILLUS OF TUBERCLE.}

BY E. W. CushINa, M.D.

Your secretary, gentlemen, has requested me to supplement my last paper before this section, in which I went over the proofs of the infectious nature of tuberculosis, by a practical demonstration of the method of staining suspected sputa for microscopic examination, with reference to bacilli.

I have accepted his invitation, at some risk of wearying you, and perhaps of being considered a bacteriomaniac, since I realize how different it is to read of a practical process, from what it is to see it done. I have still in my note-book copies of descriptions of staining for tubercle bacilli, given in 1883 and 1884, by Ehrlich, Fraenkel, Ernst, and others; but it all seemed far-off, and made no deep impression on me.

I am sure it would have aroused more interest in me, for this important matter, if $I$ had seen it done. It is curious how mythical and difficult strange chemicals seem, until one has handled them; methyl-violet seems transcendental, and vesuvin portentous, even dangerous. But when handled and used they are as commonplace as hectograph ink, or easter-egg stains.

There are many ways in which tubercle bacilli may be stained, and several in which they may be so stained as to be differentiated from all other bacilli. Some methods are easy and simple, others more complicated. In this, as in many other things, however, the easiest way is not always the best way, for it is not now a question of showing their presence when they are abundant, that is easy enough in any way: the diff-

2 The writer also desires to express his obligation to the article by Joseph Stubbs, M.D., entitled "Pneumonia treated by Venesection,",
printed in the Philadelphia Medical and Surgical Reporter, July 8, 
culty is in finding them when very few and far be- brouzed appearance of either of the above when dried tween, or in feeling certain that they are absent in a from an alcoholic solution is very beautiful. They suspicious case. For this we must use the surest both have the property of losing their color instantly method, one well proved and most likely to show all when in the presence of a strong acid, as may be seen the bacilli present.

'The orthodox method for rapid staining which is usually sufficient, and which I described in my last paper, is that with aniline-oil water, methyl-violet (or fuchsin) and nitric acid, followed by vesuvin (or methylene-blue), and of this I will show you all the steps, afterwards showing another way of making a permanent test solution of fuchsin, to be used with methylene-blue as a contrast.

'The only objection to the first method is that the solution is not permanent, it does not keep well. Koch says that it will keep about ten days, in a wellstoppered bottle, probably meaning in a laboratory where it is in daily and frequent use; I have tried keeping a solution in a dark bottle, excluding the light, and found it to keep very well for over a month.

Now first as to the materials. Aniline oil is a brown liquid which when fresh is colorless, but seems to be just as good when brown. It is a product of the destructive distillation of coal, a by-product of gas manufacture, or is made on a large scale by the action of reducing agents on nitrobenzol. The formula of aniline oil is $\mathrm{C}_{6} \mathrm{H}_{7} \mathrm{~N}$, and it may be considered as a phenyl ammonia $\left(\mathrm{C}_{6} \mathbf{H}_{5}, \mathrm{H}, \mathbf{H}\right) \mathbf{N}$., and like ammonia it forms salts with various acids.

As it was observed that bacteria took aniline dyes better when in alkaline solutions, and as in fact Koch first stained the tubercle bacilli with a solution of methylene-blue containing caustic potash, it occurred to Ehrlich to try the effect of using a solution of aniline oil instead of potash or ammonia, as the solutions of fuchsin and methyl-violet do not work well with the latter alkalies.

The results were highly satisfactory, and with some unimportant modifications Ehrlich's process has come into general use, and Koch in his last publication, ${ }^{1}$ issued in 1885 , says that he always uses it and most emphatically recommends it.

Besides aniline or aniline oil there is a similar body formed in the same way from toluol $\left(\mathrm{C}_{7} \mathrm{H}_{8}\right)$ that aniline is formed from benzol $\left(\mathrm{C}_{6} \mathrm{H}_{6}\right)$. This is called toluoidine, and may be mixed with water, filtered and used precisely like aniliue.

From mixtures of these two substances various compound salts are obtained of most remarkable colors, giving the so-called aniline dyes. These are divided by Ehrlich into acid and basic, according to whether the acid or the color-base is the coloring principle, as far as concerns the election of certain tissues or microbes for the color. The so-called acid dye-stuffs, like eosine, picric acid, alizariu, etc., do not concern us here. For staining tubercle bacilli we use exclusively the basic aniline colors.

Among these are methyl-violet (chloride of trimethyl-rosaniline) in crystals and in alcoholic solution; in the last case it is very like hectograph ink, if not identical with it. The latter fluid is said to be an excellent material for staining tubercle bacilli when it is added to water to saturation. Fuchsin (chloride of rosaniline) in small crystals, and in solution, is readily recognized as one of the dyes commonly sold to stain Easter eggs. The peculiar shining 
taken is laid between two cover-glasses, which are then rubbed together until the drop is quite evenly distributed. The glasses are then slid apart, not lifted from each other, but separated by sliding them in parallel opposite directions. They are then turned wet side up and allowed to dry thoroughly; they should meanwhile be protected from dust.

After this, one must remember which is the side with the sputum, for it is very hard sometimes to distinguish by sight the prepared side from the clean one. If necessary, the distinction is made by observing, when the glass begins to dry, which side is dull and lustreless and which is bright when so held as to reflect the light. The clean side will act like a mirror.

When dry and not sooner, the cover is to be passed three times, with the clean side down, through the flame of a Bunsen burner or spirit lamp, not too fast, nor yet slowly enough to scorch it. 'This bakes on the sputum, so that it will not wash off. 'Then it is laid in a watch-glass swimming clean side up on some of the first stain, here methyl-violet, and heated by passing back and forth through the flame, until little bubbles form under the cover-glass. That is the auspicious moment to stop the heat; in a moment it will boil up in a way that shocks the nerves, and the agitated hand scatters the stain over table and trousers, or the glass cracks, spreading dismay and sometimes causing profanity,-I would advise beginners to get a little porcelain dish, and, supporting that on a stand, to move the lamp back and forth under it; when the crisis comes, nervousness is less disastrous. The dish is now left to cool for some ten minutes - or, instead of the above heating of the fluid, the cover-glass may be left overnight to swim on the staining-fluid, without heating, say for twenty-four hours, and this is how sections of tissues are treated, because they cannot endure boiling; sections cut in celloidine must have the latter removed by ether and alcohol before staining for bacilli. For a negative diagnosis, that is, when no bacilli are found by the first process, and it is desired to make sure that none are present, it is well to leave some cover-glasses floating on the unheated staining solution from one day to another; also, when there is no hurry, this method saves trouble.

Next the cover-glass is taken up with forceps, not forgetting which is the prepared side, and, after rinsing in water, or preferably in 60 per cent. alcohol, is put in the nitric acid.

Instantly the color changes, it becomes light yellowish-brown and mostly disappears; when the color is nearly gone the glass is washed in pure water; here it will again become faintly violet, and if the color is feeble it is well ; if, however, it is strong, the glass must be dipped in the acid again. After washing, the glass is made to swim on the solution of vesuvin, (or if fuchsin has first been used, methylene-blue is the proper contrast) for about five minutes. 'This removes the remaining violet color from everything except the tubercle bacilli, and stains the pus cells and any other bacteria present in the contrasting color.

The glass is now washed in water, and may be examined in water, or dried and examined in cedar oil, or dried and mounted in Canada balsam. The latter must contain no chloroform, and is best used warm, so that when cool the preparation is firm, and will not move when rubbed to remove the oil used with the immersion lens. No oil of cloves must be used, as this will discharge the color from the bacilli; this is not important for sputa, for here the cover may be simply dried and put up in balsam; but in sections of tissues it is important to use cedar oil instead of oil of cloves for clearing up the tissues. 'The balsam must not be heated too hot; if necessary it can be thinned with oil of turpentine or xylol.

This, then, is the full and orthodox method, as successively improved by Ehrlich, Weigert, and Koch. Fraenkel puts the acid into the vesuvin (or blue) solution, thus making two steps instead of three; it is not found to be a practical advantage, however.

Of course there has been a strong desire to have a permanent solution, and it occurred to Ziel to use carbolic acid (or phenyl alcohol $\left(\mathrm{C}_{6} \mathrm{H}_{5}\right) \mathrm{H} \mathrm{O}$, instead of aniline oil (or phenyl ammonia).

His formula is as follows :

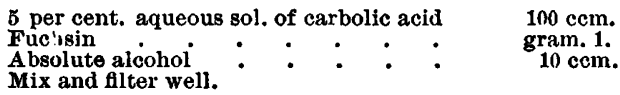

For discharging the color, muriatic acid one part, water two parts, is used. This fuchsin solution is quite permanent. I have used one solution for five months. I filtered it yesterday, but there was no sediment. It is used precisely as above, and gives excellent results. I show you specimens made last July with Liel's method, at the pathological institute in Vienna, where the energy of Professor Kundrat and the zeal of his accomplished assistants, Drs. Paltauf and Kolisko, both carry on a great amount of valuable work in bacteriology and offer great facilities for post-graduate study. The specimens have not faded at all as yet, and except for difficult diagnosis or cases where it is especially desirable to show that no bacilli are present, I can recommend the latter formula, which is quite in favor at the pathological institute in Vienna.

It is possible, but by no means certain that it is not so sure of finding isolated and rare bacilli, but, if found, the chemical reaction and diagnosis is just as perfect as by the first method given. There are other methods, but, as this is meant to be a practical demonstration and not an exhaustive paper, I will say nothing more about them.

A word as to microscopes. I have brought two, oue with the ordinary system of lenses and illumination, including a small sub-stage bull's eye condensor. The other with oil immersion and Abbé condensor. You can observe the great superiority of the latter, and yet with the former and with a power of 400 , which most microscopes give, the tubercle bacilli can be recognized.

It is fortunate that they are comparatively large, so that it is not necessary for recognizing them to have a special microscope, however desirable it may be. For those who expect to buy a microscope, for this or any other purpose, however, I would strongly recommend such a one as I show you, with a 1-12 wide angle oil immersion and an Abbé condensor; the latter is a bull's eye, flat above, about one inch in diameter, placed below the stage. It receives the light from a very large plane mirror and throws it from all sides on the highly colored bacteria, giving plenty of light with the highest powers, and, when used without diaphragms, abolishing all shadows in tissues, so that they become semi-transparent, and the bacilli can be seen below the surface of the tissue, or of the layer of sputum. With suitable diaphragms the Abbé condensor can be used 
for ordinary histological work. The ordinary size can only be adapted to microscopes with a large stage, and plenty of room, but Klonne and Müller of Berlin, (Prinzen Strasse) have arranged a system to put on common microscopes, which works very well for most purposes, and is quite sufficient for finding tubercle bacilli. The cost of a 1-12 oil immersion, like this, made by Leitz of Wetzlar, is in Germany twenty-five dollars. An Abbè condensor costs half as much; you will observe that the whole system of condensor and diaphragms can be slid up and down, by which the focus can be altered so as to give the best definition for different sources of light, objectives, or oculars; usually the upper surface of the condensor should just touch the lower surface of the slide. The oil used with the immersion is equal parts of Ol. Ricini and Ol. Foeniculi, this, being of about the same angle of refraction as glass, renders correction for thickness of cover-glass unnecessary.

Perhaps it is not superfluous to say that oil, if used with water immersion lenses, will spoil them by soaking between the glasses. The specimen which I have here stained is pus from the lung of the patient whose tuberculous larynx has just been shown you by Dr. Gannett, to whose courtesy I am indebted for the specimen of lung.

The preparation is now ready, and as the Canadis balsam was warm, it is already firm, now that it is cold. With a power of 400 you can see a large number of the bacilli, stained bright violet, on a yellowishhrown ground. With addition of a drop of oil and under the highest power of theimmersion 1-1\%, and No. 3 ocular, we get an amplification of 1,000 diameters. You can see that many of the bacilli contain spores, which appear white, as they do not take the color.

Under the next microscope is a preparation of tubercle bacilli from a specimen of sputum brought me by Dr. H. I. Bowditch.

As you see, they are very distinct, and in this place there are four or five close together, although there are only a few on each slide. One slide had none, one about ten, and one perhaps twenty. The patient was a boy, who after undergoing an attack of pulmonary disease some years ago, had had now for four weeks frothy expectoration, cough and suspicious symptoms. The results of physical examination were not sufficient, in the opinion of Dr. Bowditch, to warrant a diaguosis. There was some rough respiration in right front of chest, and an obscure and uncertain dulness, with slight bronchial respiration and crepitant râle, in lower half of same lung. Altogether, however, Dr. Bowditch felt that the physical and other symptoms were so indistinct that no positive diagnosis could have been made except by finding the bacilli.

I have also here a similar preparation from sputum, sent me by Dr. Marcy, of a gentleman whose rational and physical symptoms were too equivocal to warrant a diagnosis. There was little expectoration, once slightly tinged with blood: he was in middle life, not emaciated, very active and vigorous, and attending to his business. There was a slight diminution of respiratory force at the right apex ; expiration slightly prolonged; no crepitant râles.

The bacilli, though infrequent, are very distinct, and the decision by the microscope has caused the patient, by the advice-of Dr. Marcy, to decide to make arrangements to go and live for a long period in a more favorable climate.
Per contra, in a specimen sent me by Dr. Knight, from a patient whose symptoms, though indefinite, were very suspicious - particularly an erosion of each vocal chord, forming in each a deep notch - I was unable to find any bacilli.

Dr. Knight stated that these deep erosions or nicks in the chords were, in his experience, seldom found, except in cases where tuberculosis was present, but here the examination of the chest gave no diagnosis, although the expectoration and the condition of the chords gave ground for anxiety.

The patient's condition improved for a time, but three months after the above examination he grew worse, with high temperatures, etc., but no clear physical signs. I now found plenty of bacilli in the sputa. He died a few weeks later.

In this bottle I have sputum sent me by Dr. Clarke, of Cambridge, from a case similar to the one of Dr. Marcy's above mentioned, where the principal symptom is profuse expectoration in a gentleman who is able to attend to his business, and has no physical symptoms from which a diagnosis can be made, although his general condition is sufficient to cause some anxiety. Here a very few bacilli are to be found, two or three on each cover-glass.

The above cases are fair samples of others which I could mention, showing the advantage of microscopic examination of the expectorations of patients even suspected of being consumptive. Of course, in well-developed cases, the disease can be realily recognized by other means of examination, such as auscultation and percussion. But in some cases, it is certain that the diagnosis can be made by the microscope several, or many, months before the disease is sufficiently developed to be diagnosticated in any other way. Who shall measure the advantages of an early discovery of such a fatal disease, in those who, by a change of climate, occupation, etc., are able to put themselves under the best conditions for getting well?

It is for this reason that $I$ insist on the importance for practical physicians, of this method of diagnosis. I wish to compare it, in importance and usefulness, with the examination of the urine for casts in Bright's disease. Some cases of Bright's do not show casts during considerable periods; in some cases, the diagnosis can be made pretty surely without the microscope. Not every one can easily tell a cast from a roll of mucus with cells in it. But yet no one would say that he had made a real and proper examination of a patient with suspicious symptoms, until the urine had been well searched for casts.

Now, mutatis mutandis, all this is true of phthisis pulmonum, and my contention is that it is the duty of a physician who undertakes to make a diagnosis of the presence or absence of consumption, to examine the sputa for bacilli, except in cases so far advanced that there is no difficulty whatever by ordinary methods. It is not so very long since ordinary methods, namely, auscultation and percussion, were considered new-fangled and unnecessary ; in proof, the funny verses of our venerable poet-physician, who, I believe, brought one of the first stethoscopes to Boston, at a time when the lights of those times could, as they thought, make a diagnosis easily enough from the general symptoms.

The examination of sputa by the microscope for bacilli is now taught to the students at Harvard; it is learned by those who go abroad, where they see it 
used, as a matter of course, in the hospitals in all cases suspected of consumption.

Sometimes the diagnosis is difficult ; careless work, or poor instruments, may make it erroneous; but the same is true of Bright's disease, and in fact, of most other diseases.

The method is not a mere craze; it has come to stay, and any one of the profession who wishes to be teres, totus atque rotundus, to do his very best by his cases, ought to take it up, learn it, and use it. I shall be very glad if $I$ have here been of any service to such.

As a matter of convenience, in recording an approximate estimate of the number of bacilli found, in the average of slides examined in any case, $I$ subjoin the scale given by Gaffky, in the Communications of the German Imperial Health Office, 1884. He divides the numbers of bacilli found on one cover-glass, prepared as above, into ten grades, as follows (it being understood that the field visible at any one time is that seen with objective 1-12 oil immersion, ocular II, giving a power of 800 diameters ; the area seen is about 1-4 millimeter, or 1-100 inch in diameter) :

1. In the whole amount on a cover-glass, 1-4 bacilli.

2 . In several microscopic fields of view together, only 1 bacillus.

3. In every microscopic field of view, on the average, about 1 bacillus. 4 . " " " "

5. 6 " 6

8.

10.

" $\begin{array}{r}4-6 \\ 7-12\end{array}$

a good many

" numerous

" very numerous "“

bacilli.
normous numbers of

I think this scale convenient, and as nearly accurate as any method of recording or comparing can be, and, if generally adopted and understood, it will be found useful in recording examinations, and in comparing the condition of the patient at different times.

It is not necessary that the sputa be examined at once when collected. The bacilli stand ordinary putrefactive changes in their environment very well. The sputa may be kept or sent from one place to another either in a vial, or by being dried and baked on to cover-glasses, as I have showed you to-night. The addition of a weak solution of carbolic acid does not interfere with the coloring, but is not necessary, except to prevent unpleasant odors.

In reply to the question as to what amount of examination seems necessary in order to say that the bacilli are not present, I would say that, in a doubtful case, I think it sufficient to prepare three pairs of coverglasses, using the quick method with two pairs, and leaving the other two glasses to swim, until the next day, on the unheated staining fluid. If no bacill are found in the six glasses thus examined, another examination, or even two, should be made at intervals, just as is customary in searching for casts in Bright's disease.

If still no bacilli are found, it is pretty safe to infer that the patient has not, at that time, pulmonary tuberculosis (unless it be acute miliary), although theoretically, it is possible that the bacilli may be in the lungs, and have no access to the bronchi; but for ordinary phthisis, or consumption, the diagnosis can be made with practical accuracy.

Hotel Pelham, Boston.

- Russia has 33,400 physicians, of whom 380 are females. There are 2,100 persons.licensed to practice veterinary surgery, 500 are enrolled as dentists and " oculists."

\section{REPORT ON MEDICAL CHEMISTRY.}

\author{
BY WILliaM B. HILLS, M.D.
}

ARSENIC.

IN cases of suspected arsenical poisoning, it is sometimes a question whether any poison found was taken into the body during life or was introduced after death. This question arose in a case which was tried a few years since in Michigan. ${ }^{1}$ A lady died with symptoms which strongly suggested irritant poisoning. The body was exhumed one hundred and five days after death. Prof. A. B. Prescott found about twenty grains of arsenious oxide in the stomach and rectum together, and he calculated from an analysis, that the whole liver contained from six to fifteen grains, according to the size of the organ. 'The body was again exhumed later, and the brain and a portion of the muscles of the calf of the leg examined; in these no arsenic was found. The husband claimed to have injected arsenious oxide suspended in water into the mouth and rectum for the purpose of preserving the body. The six experts in the case were equally divided on the question "granting that the arsenic was injected into the mouth and rectum in the manner claimed, could it reach the liver and other organs outside the alimentary canal ?"

Drs. Vaughan and Dawson made some experiments at this time, which showed that, when arsenious oxide was injected into the mouth and rectum after death, it was so far diffused that at the end of twenty-five days it could be detected in the liver, heart, spleen, kidneys and brain.

More recently, Mr. Frank S. Sutton has made a series of experiments on this subject in the laboratory of Professor Wormley, the bodies of dogs killed with chloroform being employed. ${ }^{2}$ In each case three grammes of arsenious oxide suspended in fifty cc. of water were injected into the stomach, and an equal quantity of the oxide in ten cc. of water into the rectum. The body was then buried in the earth. In four cases, in which the injections were made in from twenty-three to twenty-six and one-half hours after death, and the bodies exhumed at the end of one hundred and two, seventy-four, forty-four and eighteen days respectively, arsenic was found in the liver, kidneys and brain. The quantity of arsenic was larger the longer after the introduction of the poison the body had been buried. In the most protracted case ten milligrammes of arsenious oxide were found in the liver.

Even when the injection was made ten minutes after death and the body examined at the end of three days, the final solution from the brain gave a wellmarked stain in the reduction-tube of a Marsh apparatus, and satisfactory evidence of the presence of the metal in the liver and kidneys was obtained. But when the injection was made twenty-four hours after death and the body examined at the end of three days, the final solution from the brain furnished only the faintest trace of arsenic. The metal was present in very minute quantity in the liver, but it seemed to be entirely absent from the -kidneys. These experiments show that, when the injection is made very soon after death, the diffusion takes place more readily than when the injection is not made until some hours after death.

This question of the post-mortem diffusion of arsenic

1 American Journal of Medical Sciences, October, 1883, page 599.

2 Wormley's Micro-Chenistry of Poisons, Second Edition, page ¿14. 